\title{
Single case experimental design
}

David F Marks

Potential competing interests: The author(s) declared that no potential competing interests exist.

Single case experimental designs are investigations of a series of experimental manipulations with a single research participant. 